\title{
Changes in Diagnosing Non-ST-Segment Elevation Myocardial Infarction after the Introduction of a New High-Sensitivity Cardiac Troponin T Assay: A Single-Centre Experience
}

\author{
TRYGVE SØRDAHL HALL ${ }^{1,3}$, JONAS HALLÉN ${ }^{2,3}$, STEFAN AGEWALL ${ }^{2,3}$, \\ DAN ATAR ${ }^{2,3}$, TORSTEIN JENSEN $^{1}$ \\ ${ }^{\prime}$ Department of Cardiology, Lovisenberg Diakonale Hospital, Oslo, Norway \\ ${ }^{2}$ Department of Cardiology, Oslo University Hospital, Oslo, Norway \\ ${ }^{3}$ Institute of Clinical Medicine, University of Oslo, Oslo, Norway
}

\begin{abstract}
SUMMARY
Background: Cardiac troponins are the most sensitive and specific biochemical markers of myocardial injury and with the new high-sensitivity troponin methods very minor injuries of the heart muscle can be detected. The introduction of high-sensitivity assays has facilitated reference range adjustments and a revised cut-off point for myocardial infarction (MI) due to an improved performance in the lower concentration range. The objective of this study was to investigate whether implementing a high-sensitivity cardiac troponin $T$ (hs-cTnT) assay with subsequent lowering of the cut-off point changed the hospital evaluation and diagnosis of acute non-ST-segment elevation myocardial infarction (NSTEMI) in a general hospital population.

Methods: NSTEMI patients admitted to our hospital during two periods each lasting one year were retrospectively compared. During period 1 (August 2007 - July 2008) patients were diagnosed with a conventional troponin $T$ assay, and during period 2 (August 2009 - July 2010) patients were diagnosed using an hs-cTnT assay.

Results: A significant increase in the number of NSTEMI admissions was observed using the hs-cTnT assay (225 vs. 341 , risk ratio $1.57,95 \%$ confidence interval 1.33 to 1.85 ). The proportion of patients examined with acute coronary angiography was similar $(\mathbf{2 5 . 8} \%$ vs. $\mathbf{2 3 . 8 \%})$. Due to the higher number of NSTEMI admissions the total number of angiographies was higher in period $2(58 \mathrm{vs} .81, \mathrm{p}<0.05)$, and significantly more patients were examined without signs of coronary artery disease (CAD) $(0 \%$ vs. $8.6 \%, p<0.05)$. A smaller proportion diagnosed with the high-sensitivity assay had significant dynamic cTnT changes between the highest and lowest cTnT measurement during each admission $(96.2 \%$ vs. $88.7 \%$, p $<0.01)$.

Conclusions: More patients were diagnosed with NSTEMI and underwent coronary angiography after introducing the hs-cTnT assay. At the same time there was an increase in the frequency of coronary angiograms without signs of CAD, and fewer had significant dynamic cTnT concentration changes.

(Clin. Lab. 2012;58:1029-1036. DOI: 10.7754/Clin.Lab.2012.111206)
\end{abstract}

\section{KEY WORDS}

NSTEMI, MI, ACS, hs-cTnT, cTnT, MINCA

\section{INTRODUCTION}

Cardiac troponins (cTn) are myocardial contractile proteins. The cTn complex includes troponin subtypes $\mathrm{T}$ (cTnT), I (cTnI), and C. They facilitate cross-bridging of actin and myosin filaments. CTnT and cTnI are re- garded as specific to myocardial tissue [1] and have been established as biomarkers of choice for myocardial injury [2-4].

In the consensus statement "Universal definition of myocardial infarction" it is recommended that the 99th percentile concentration value of cTn in a normal reference population should be used as a cut-off point for diagnosing myocardial infarction (MI) [2]. The use of assays without optimal precision (a coefficient of varia-

Manuscript accepted January 18, 2012 
tion (CV) of less than $10 \%$ ) at the 99th percentile is discouraged, and thus some have advocated the use of a higher cut-off point if a $10 \% \mathrm{CV}$ cannot be achieved at the 99th percentile [5]. Conventional cTn assays are imprecise at very low levels, whereas new hs-cTn assays have shown improved sensitivity and less variation $[6$, 7]. Due to the varying performance of cTn assays different cut-off points have been used in clinical practice [8]. Upon introduction of hs-cTnT in April 2009, our hospital adjusted the cut-off point for MI from $100 \mathrm{ng} / \mathrm{L}$ to $30 \mathrm{ng} / \mathrm{L}$ according to national guidelines based on the "Universal definition of myocardial infarction".

The introduction of the hs-cTnT assay and the lower cut-off point was expected to result in more patients qualifying for the biomarker criterion for MI. Previous studies have documented that the application of a highsensitivity cTn assay and a subsequent lower cut-off point increased the proportion of patients diagnosed with MI in suspected acute coronary syndrome $[9,10]$ and in unselected patients presenting to the emergency department with chest pain [11].

The aim of this study was to investigate whether an increased number of non-ST-segment elevation myocardial infarction (NSTEMI) would be observed in an unselected patient population by considering all patients that had been admitted to the medical department in two one-year periods. Furthermore, coronary angiograms were registered to investigate possible changes in the frequency of examinations without coronary artery disease (CAD). We also evaluated whether there were differences on the fulfilment of the cut-off point criterion and described the dynamics of cTnT measurements performed during these admissions.

\section{MATERIALS AND METHODS}

\section{Study population}

The study population comprised all patients admitted to the medical department of Lovisenberg Diakonale Hospital, which serves as the acute medical care facility for adults in four different city areas of Oslo, Norway. The hospital has approximately 6500 admissions during one year. We compared two one-year periods: Period 1 (p1) from $1^{\text {st }}$ of August 2007 to $31^{\text {st }}$ of July 2008 (catchments population 145000 people), and period 2 (p2) from $1^{\text {st }}$ of August 2009 to $31^{\text {st }}$ of July 2010 (catchments population 155000 people). The hs-cTnT assay was introduced on the $1^{\text {st }}$ of April 2009. All coronary angiographies were performed at a secondary care facility (Oslo University Hospital) upon referral when deemed indicated by the clinician.

Discharge papers were coded by the treating physician using ICD-10. The appropriate diagnoses were registered together with other relevant clinical information allowing for retrieval of all MI admissions (ICD-10 code I21 and I22). Inclusion criteria were all admissions with a registered diagnosis of MI categorized as a primary NSTEMI admission. A primary admission was defined as a patient admitted directly from the ambulance service, emergency department, outpatient clinic, department of surgery or general practitioner. All other stays such as transfers from other hospitals, re-admissions after coronary angiography investigations and others were excluded. Electrocardiography (ECG) recordings were reviewed on all admissions to include only NSTEMI patients.

\section{Registration of data}

All MI admissions with associated demographic information and measured cTnT values were retrospectively retrieved from the hospital database by a blinded physician using Qlikview (Qlik Technologies Inc., Radnor, Philadelphia, PA, USA). The authors then reviewed this information, along with patient charts, ECG recordings, and angiography reports, using the DIPS electronic medical record system (DIPS ASA, Bodø, Norway). Appropriate variables were registered in the PASW Statistics 18 program (IBM SPSS Inc., Chicago, IL, USA). All definitions used in the data registration were predefined in accordance with international practice guidelines. Angiographies were defined as acute if the patients were transferred directly from our hospital to the secondary care facility following a primary NSTEMI diagnosis, and the results were classified using prespecified criteria by evaluating the written reports from these examinations (Table 3 ). The highest cTnT level was defined as the highest concentration of measured cTnT during the hospital stay (Table 2). To describe cTnT dynamics we defined the relative change to be calculated using the lowest registered measurement before and after the highest concentration. These were then categorized into groups to allow comparison (Table 2). Admissions with $<2$ cTnT measurements did not have a relative change calculated.

\section{The cTn analyzer}

An electrochemiluminescent immunoassay was used for the analysis of cTn. The conventional assay used during p1 was Elecsys ${ }^{\circledR}$ Troponin T on Modular Analytics E 170 by Roche Diagnostics (Roche, Basel, Switzerland). The new assay used during p2 was Elecsys ${ }^{\circledR}$ hs TnT on the same analyzer. For the old assay, the manufacturer designates a limit of quantification (lowest concentration that can be reproducibly measured with a betweenrun $\mathrm{CV}$ of $\leq 10 \%$ ) for the conventional assay to $30 \mathrm{ng} / \mathrm{L}$ and a 99th percentile cut-off point of less than $10 \mathrm{ng} / \mathrm{L}$. For the new assay the manufacturer reports a limit of quantification at $13 \mathrm{ng} / \mathrm{L}$ and a 99th percentile cut-off point at $14 \mathrm{ng} / \mathrm{L}$. During in-house quality-control analyses, our laboratory found a CV of $8.3 \%$ at a concentration of $72 \mathrm{ng} / \mathrm{L}$ with the conventional assay and a $\mathrm{CV}$ of $4.7 \%$ at a concentration of $34 \mathrm{ng} / \mathrm{L}$ with the new assay. As mentioned above, the cut-off point for MI in our hospital was $100 \mathrm{ng} / \mathrm{L}$ in $\mathrm{p} 1$ and $30 \mathrm{ng} / \mathrm{L}$ in $\mathrm{p} 2$. 
Table 1. Baseline characteristics.

\begin{tabular}{|c|c|c|}
\hline \multicolumn{3}{|c|}{ All medical department admissions } \\
\hline Variable & $P 1(n=6704)$ & $P 2(n=6486)$ \\
\hline Different individuals, $n$ & 4401 & 4260 \\
\hline Males, $\%$ & 46.3 & 48.4 \\
\hline \multicolumn{3}{|l|}{ Age (years), mean (SD) } \\
\hline Males & $59.2(18.8)$ & $59.8(18.5)$ \\
\hline Females & $65.6(22.7)$ & $65.4(22.6)$ \\
\hline Length of stay (days), median (quartile 1 to 3 ) & $3(0-7)$ & $3(1-7)$ \\
\hline Primary NSTEMI admissions, $\mathrm{n}(\%)$ & $225(3.36)$ & $341(5.26)$ * \\
\hline Acute angiographies due to NSTEMI, n (\%) & $58(0.87)$ & $81(1.25) *$ \\
\hline \multicolumn{3}{|c|}{ Primary NSTEMI admissions } \\
\hline Variable & P1 $(n=225)$ & $P 2(n=341)$ \\
\hline Different individuals, $\mathbf{n}$ & 202 & 300 \\
\hline Males, $\%$ & 48.4 & 44.6 \\
\hline \multicolumn{3}{|l|}{ Age (years), mean (SD) } \\
\hline Males & 71.9 (13.8) & 72.7 (13.5) \\
\hline Females & $81.4(12.3)$ & $83.8(11.3)$ \\
\hline Length of stay (days), median (quartile 1 to 3 ) & $7(2-12)$ & $6(3-11)$ \\
\hline Angiographies, $\mathbf{n}(\%)$ & $58(25.8)$ & $81(23.8)$ \\
\hline
\end{tabular}

* Indicates a statistically significant difference $(p<0.05)$.

Table 2. Evaluating the cut-off criterion and dynamic cTnT changes.

\begin{tabular}{|c|c|c|}
\hline Type & P1 $(\mathrm{n}=225)$ & P2 $(\mathrm{n}=341)$ \\
\hline Cut-off criterion fulfilled, $\mathbf{n}(\%)$ & $201 / 225(89.3)$ & $335 / 341(98.2) *$ \\
\hline $\begin{array}{c}\text { CTnT not measured or in normal } \\
\text { reference range, } \mathbf{n}(\%)\end{array}$ & $5 / 225(2.2)$ & $1 / 341(0.3)$ \\
\hline $\begin{array}{c}\text { Highest cTnT measured below cut-off, but above normal } \\
\text { reference range, } \mathbf{n}(\%)\end{array}$ & $19 / 225(8.4)$ & $5 / 341(1.5) *$ \\
\hline Relative change $\geq 20 \%, n(\%) * *$ & $204 / 212(96.2) *$ & $290 / 327(88.7)$ \\
\hline
\end{tabular}

* Indicates a statistically significant difference $(\mathrm{p}<0.01)$ ** 13 admissions in $\mathrm{p} 1$ and 14 admissions in p2 excluded since $<2$ cTnT measurements performed on these admissions.

\section{Ethical considerations}

The study was conducted according to the principles of the Declaration of Helsinki and the Uniform Requirements for Manuscripts Submitted to Biomedical Journals by the International Committee of Medical Journal Editors. It was approved by our hospital's local ethics committee and the privacy protection act supervisor.

\section{Statistical analysis}

Categorical variables are presented as numbers and/or percentages and continuous variables are presented as means (standard deviation (SD)) or medians (quartiles 1 to 3). Continuous variables were compared with the $t$ test (if normally distributed) or the Mann-Whitney U test (if skewed). Categorical variables were compared using the Pearson chi square test or Fischer's exact test (if any field included $<6$ ). Some individuals had more than one admission, and the total number of admissions is therefore higher than the number of individuals. In the statistical analysis the number of admissions has been used for comparison of variables. A p-value of $<$ 0.05 was regarded statistically significant, and all hypothesis testing was two tailed. 
Table 3. Coronary Angiography.

\begin{tabular}{|c|c|c|}
\hline Variable & $P 1(n=58)$ & $P 2(n=81)$ \\
\hline \multicolumn{3}{|c|}{ Findings * } \\
\hline No CAD & O (0) & $7(8.6) * *$ \\
\hline Insignificant/borderline & $4(6.9)$ & $5(6.2)$ \\
\hline 1-vessel disease & $25(43.1)$ & $27(33.3)$ \\
\hline 2-vessel disease & $18(31.0)$ & $22(27.2)$ \\
\hline 3-vessel disease & $8(13.8)$ & $14(17.3)$ \\
\hline Left main stem (LMS) + others & $3(5.2)$ & $6(7.4)$ \\
\hline \multicolumn{3}{|c|}{ Interventions performed } \\
\hline BMS & $25(43.1) * *$ & $13(16)$ \\
\hline DES & 7 (12.1) & $25(30.9) * *$ \\
\hline Any stent & $32(55.2)$ & $38(46.9)$ \\
\hline CABG & $6(10.3)$ & $9(11.1)$ \\
\hline BMS and DES & $2(3.4)$ & $0(0)$ \\
\hline None & $18(31.0)$ & $34(42.0)$ \\
\hline \multicolumn{3}{|c|}{ Prior interventions } \\
\hline Stent & $5(8.6)$ & $18(22.2) * *$ \\
\hline CABG & $1(1.7)$ & $2(2.5)$ \\
\hline Stent and CABG & $2(3.4)$ & $4(4.9)$ \\
\hline None & $50(86.2) \div *$ & $57(70.4)$ \\
\hline
\end{tabular}

Data presented as $\mathbf{n}(\%)$. * Lesions in coronary bypass grafts were not evaluated in the study. ** Indicates a statistically significant difference $(\mathbf{p}<0.05)$.

Description of groups and intervention types in Table 3:

Variable

Findings

No CAD

Insignificant/borderline

1-vessel disease

2-vessel disease

3-vessel disease

Left main stem (LMS) + others

Interventions performed

BMS

DES

Any stent

CABG

BMS and DES

None

Prior interventions

Stent

CABG

Stent and CABG

None

\section{Description}

No pathology in any vessel

Changes present but no changes $>\mathbf{5 0} \%$

$>\mathbf{5 0} \%$ in 1 vessel

$>\mathbf{5 0} \%$ in 2 vessels

$>\mathbf{5 0} \%$ in 3 vessels

Change in LMS + others

\section{Bare-Metal Stent}

Drug-Eluting Stent

DES or BMS

Coronary Artery Bypass Graft

BMS and DES

No intervention

Prior stent in situ

Prior CABG in situ

Prior stent and CABG in situ

No prior intervention

\section{RESULTS}

Demographics and baseline characteristics

Table 1 shows demographic characteristics of the hospital's patients in the two periods. The medical department had a total of 6704 admissions in p1 (4401 individuals), and of these $3.36 \%$ (95\% confidence interval
(CI) 2.95 to 3.81$)$ were categorized as primary NSTEMI admissions $(n=225)$. In $\mathrm{p} 2$ the total number of admissions was 6486 (4260 individuals), of which $5.26 \%$ (95\% CI 4.74 to 5.83 ) were categorized as primary NSTEMI admissions $(n=341)$. The number of NSTEMI patients was significantly higher in $\mathrm{p} 2(\mathrm{p}<$ $0.001)$, with a risk ratio of 1.57 (95\% CI 1.33 to 1.85 ). 


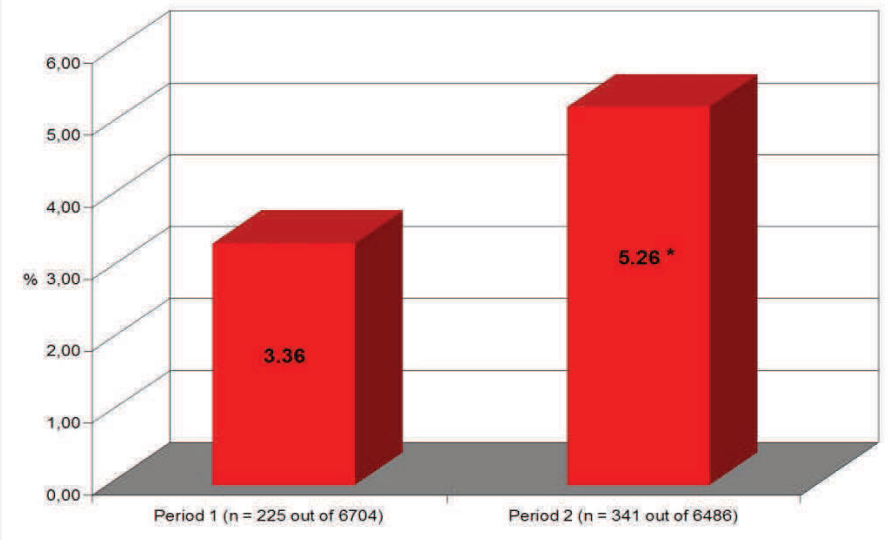

$* \mathrm{p}<0.001$

Figure 1. Proportion diagnosed as NSTEMI (\% of total number of admissions).

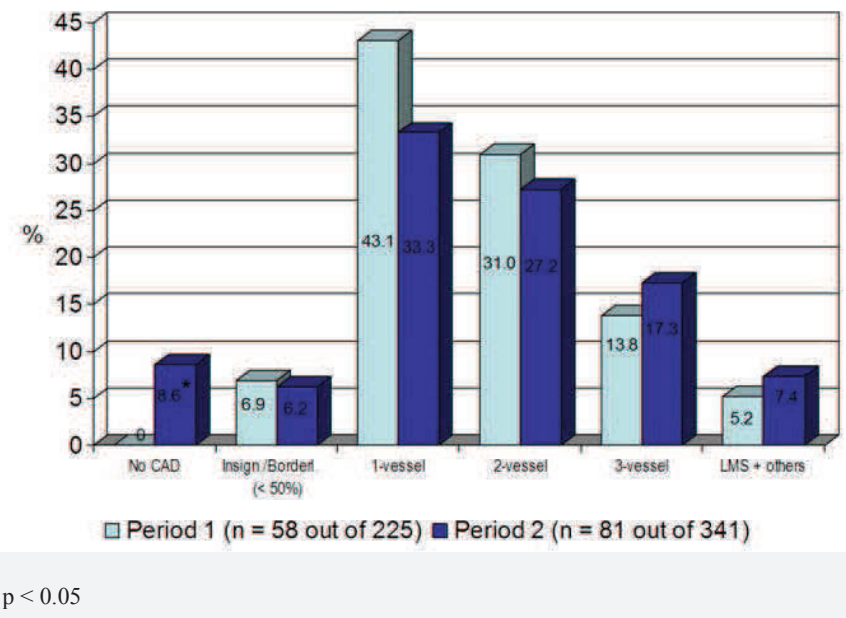

Figure 2. Angiography results (\% of total number of angiographies). 


\section{Coronary angiography}

The percentage of NSTEMI patients examined with acute coronary angiography was similar in the two periods (58 out of $225(25.8 \%)$ in p1 vs. 81 out of 341 $(23.8 \%)$ in $\mathrm{p} 2)$. The risk ratio for angiography was 1.44 (95\% CI 1.03 to 2.02 ) in $\mathrm{p} 2$ due to the higher proportion of NSTEMI admissions. The number of examinations without angiographic signs of CAD were significantly higher in this period $(0 \%$ vs. $8.6 \%, p<0.05)$, and there was also a trend of more patients not receiving an intervention ( $31.0 \%$ vs. $42.0 \%, \mathrm{p}=0.19)$.

\section{Evaluation of the cTnT measurements}

As shown in Table 2, the number of admissions fulfilling the defined cut-off criterion used in our hospital in each of the respective periods was higher when using the hs-cTnT assay $(89.3 \%$ in p1 $(100 \mathrm{ng} / \mathrm{L})$ vs. $98.2 \%$ in p2 $(30 \mathrm{ng} / \mathrm{L}), \mathrm{p}<0.001)$. Out of the $10.7 \%$ in $\mathrm{p} 1$ and $1.8 \%$ in $\mathrm{p} 2$ that did not fulfil the cut-off criterion, 19 and 5 , respectively, had elevated levels measured below the defined cut-off concentration. Five in $\mathrm{p} 1$ and 1 in $\mathrm{p} 2$ did not have a cTnT concentration analysed or measured in the normal range due to various circumstances, such as death before blood had been drawn or acute transfer to another hospital. A smaller proportion in $\mathrm{p} 2$ had relative concentration changes $\geq 20 \%(96.2 \%$ in $\mathrm{p} 1$ vs. $88.7 \%$ in $\mathrm{p} 2, \mathrm{p}<0.01)$.

\section{DISCUSSION}

In the present study, we evaluated changes in hospital evaluation and the diagnosis of NSTEMI in a general hospital population after the introduction of a new hscTnT assay. The main finding was an increased number of NSTEMI patients. We also found that more coronary angiographies had been performed, and that a larger proportion of these were without angiographic signs of CAD. Finally, more admissions fulfilled the cut-off criterion, but fewer had significant dynamic cTnT changes when evaluated by the highest and lowest measurement during each admission.

By lowering the cut-off point and thereby the detection of myocardial cell injury, more NSTEMI diagnoses are to be expected [9-12]. Our study confirmed these findings in an unselected hospital population. An additional explanation for the increase observed in $\mathrm{p} 2$ is the difference in assay calibration between the new high-sensitivity assay and the conventional assay, especially in the lower end of the measuring range. Saenger et al. showed a substantial positive bias of $76 \%$ when comparing the hs-cTnT with the conventional cTnT assay in concentrations $<50 \mathrm{ng} / \mathrm{L}$ [13], implying that measurements cannot be directly compared as the high-sensitivity assay uniformly will show higher values. Patients that previously had measurements below the cut-off point for MI can now be evaluated differently as they will have higher maximum concentrations when using the high-sensitivity assay. Thus, solely on the basis of altered assay characteristics, the introduction of hscTnT will result in more patients qualifying for a NSTEMI diagnosis at a certain cut-off level.

Elevated cTn levels can be found in a number of different clinical settings, and NSTEMI is just one of many differential diagnoses that need to be considered $[3,14-$ 16]. The improved sensitivity that followed the introduction of the high-sensitivity assays also reduced its clinical specificity $[6,7]$. A positive predictive value (PPV) as low as $50 \%$ was shown with one of the new assays [7], and in a similar study the specificity was reduced from $97.2 \%$ to $90.2 \%$ [6]. These data underscore the need for meticulous clinical evaluation, as one cannot rely solely on laboratory data in the diagnostic process.

Due to the increased number of patients that today's clinicians must evaluate triggered by an elevated level of cTnT, one could hypothesize that more patients would be misdiagnosed using the high-sensitivity assay. At the same time, we expected that more patients would be examined with angiography if the number of NSTEMIs increased. Indeed, our results show that a similar proportion of patients was referred to angiographic examination in both periods, and therefore the number of angiographies was higher in $\mathrm{p} 2$. In further reviewing the angiograms, we observed more patients diagnosed with the hs-cTnT assay who were without angiographic signs of CAD. Some of these patients may have been misdiagnosed, but there is an increasing interest and awareness of a subgroup of patients with MI classified as "Myocardial Infarction with Normal Coronary Arteries" (MINCA) [17]. The number is small, and in both periods the majority of patients that was sent to angiography did have significant disease. Taken together, we consider the new cut-off point advantageous since a larger number of patients in $\mathrm{p} 2$ was identified and subsequently referred to a higher level of care. This most likely will result in reductions in morbidity and mortality [9].

When evaluating the biomarker criteria applicable in each period, a higher proportion of patients fulfilled the defined cut-off point for MI using the hs-cTnT assay. Our hospital guideline recommended a higher cut-off level than the 99th percentile in both periods of the study. Aakre et al. have demonstrated a discrepancy between the cut-off points used in Norwegian hospitals, possibly due to varied $\mathrm{CV}$ performance at the lower concentration range [8]. Norwegian guidelines currently advocate $30 \mathrm{ng} / \mathrm{L}$ as an appropriate cut-off criterion for the hs-TnT assay, but due to the improved accuracy documented in large multicenter studies this recommendation may change $[13,18]$. Our study has shown that a few NSTEMI admissions did not adhere to the cut-off criterion recommended in the hospital guidelines. Most of these fulfilled the 99th percentile defined in the consensus statement, indicating that the treating physicians choose to adapt to this recommendation. Interestingly, we also demonstrated that a higher number of patients in $\mathrm{p} 2$ showed smaller dynamic cTnT 
changes. This may imply that more patients diagnosed in this period lacked a significant rise and/or fall pattern, which, according to the guidelines, is a prerequisite for diagnosis [2]. It has not been specifically defined yet which net change that can be regarded as a true change between two biomarker measurements [2]. Indeed, the extent of dynamic change of cTn necessary for the diagnosis is a matter of ongoing debate [19-22]. Lately the use of absolute instead of relative changes has been advocated since it may improve sensitivity and specificity [23]. The biological variation is also important to consider when interpreting laboratorial results, as especially in the lower reference range, it will contribute to different concentration values that are naturally occurring and not ischemic in origin [24-26]. In our study a $\geq 20 \%$ relative concentration change was chosen since it has been described as an appropriate concentration change to apply in these situations [10,24,27], while others have advocated to use a higher differential [28]. These studies applied serial measurements taken a few hours after admission when calculating their relative change values, while in our study we used the highest and lowest registered values on each admission without considering the timeline between these measurements. At the present time, there is no consensus as to which dynamic change can be interpreted to represent ischemic origin with certainty.

\section{Study limitations}

The observational and retrospective nature of the analyses should restrict interpretation of our findings. The inclusion criterion was based on the retrieval of patients that were registered in the database upon discharge, and it is possible that some admissions were not included due to miscoding. The physicians may have been more aware of measuring cTn concentrations in p2 since the high-sensitivity assay with a revised cut-off point had been recently introduced. Differences in baseline and follow-up confounding factors in the two periods were not investigated. In addition, a rise and/or fall pattern is not absolutely required if the patient presents $>24$ hours after the onset of symptoms [2], and this data was not extracted from the database. It may be that a higher proportion would not qualify for the $\geq 20 \%$ relative change criterion if a time limit was defined between the two values used, and some of the values used may have had more hours between them than what would be appropriate when calculating dynamic changes. Lastly, the number of patients examined with angiography is quite low, and the results must therefore be looked at with some caution. We believe that this is related to the retrospective design of an unselected patient population, with a large proportion of older patients with co-morbidity contraindicating angiographic examination.

\section{CONCLUSION}

We found that the proportion of admissions with NSTEMI in our patient population increased following the introduction of the hs-cTnT assay with an associated lower cut-off point. The number of patients investigated with coronary angiography was also higher. There was an increase in the frequency of coronary angiograms without signs of CAD, and a smaller proportion had significant dynamic cTnT concentration changes. In conclusion, our data suggest that introduction of the hscTnT assay has improved NSTEMI diagnostics, but more patients must be considered to have increased cTnT levels from other aetiologies than myocardial ischemia.

\section{Acknowledgement:}

The authors wish to acknowledge Einar Amlie for retrieving the information from the hospital database and Christian Hall for discussions and advice.

\section{Author contributions:}

Data collection: Hall TS, Jensen T; drafting article: Hall TS; concept/design/dataanalysis/interpretation/statistics/ critical revision of article/approval of article: Hall TS, Hallén J, Agewall S, Atar D, Jensen T.

\section{Declaration of Interest:}

$\mathrm{JH}$ is a Ph.d. student at Oslo University Hospital and University of Oslo, but is also an employee of Boehringer Ingelheim Norway KS. The other authors have no disclosures.

\section{Source of Support:}

The study was supported by Lovisenberg Diakonale Hospital's research fund. The research fund had no other involvement in the study.

\section{Disclaimers:}

None.

\section{References:}

1. Morrow DA, Cannon CP, Jesse RL, et al. National Academy of Clinical Biochemistry Laboratory Medicine Practice Guidelines: clinical characteristics and utilization of biochemical markers in acute coronary syndromes. Clin Chem 2007 Apr;53(4):552-74.

2. Thygesen K, Alpert JS, White HD. Universal definition of myocardial infarction. J Am Coll Cardiol 2007 Nov 27;50(22):217395.

3. Jaffe AS, Babuin L, Apple FS. Biomarkers in acute cardiac disease: the present and the future. J Am Coll Cardiol 2006 Jul 4; 48(1):1-11. 
4. Atar D. New definition of myocardial infarction. BMJ 2008; 337:a3078.

5. Apple FS, Wu AH, Jaffe AS. European Society of Cardiology and American College of Cardiology guidelines for redefinition of myocardial infarction: how to use existing assays clinically and for clinical trials. Am Heart J 2002 Dec;144(6):981-6.

6. Keller T, Zeller T, Peetz D, et al. Sensitive troponin I assay in early diagnosis of acute myocardial infarction. N Engl J Med 2009 Aug 27;361(9):868-77.

7. Reichlin T, Hochholzer W, Bassetti S, et al. Early diagnosis of myocardial infarction with sensitive cardiac troponin assays. $\mathrm{N}$ Engl J Med 2009 Aug 27;361(9):858-67.

8. Aakre KM, Landaas S, Hagve TA. [Use of troponin analysis in Norwegian hospitals]. Tidsskr Nor Laegeforen 2010 Feb 11; 130 (3):278-81

9. Mills NL, Churchhouse AM, Lee KK, et al. Implementation of a sensitive troponin I assay and risk of recurrent myocardial infarction and death in patients with suspected acute coronary syndrome. JAMA 2011 Mar 23;305(12):1210-6.

10. Giannitsis E, Becker M, Kurz K, Hess G, Zdunek D, Katus HA. High-sensitivity cardiac troponin $\mathrm{T}$ for early prediction of evolving non-ST-segment elevation myocardial infarction in patients with suspected acute coronary syndrome and negative troponin results on admission. Clin Chem 2010 Apr;56(4):642-50.

11. Christ M, Popp S, Pohlmann H, et al. Implementation of high sensitivity cardiac troponin T measurement in the emergency department. Am J Med 2010 Dec;123(12):1134-42.

12. White HD, Chew DP. Acute myocardial infarction. Lancet 2008 Aug 16;372(9638):570-84.

13. Saenger AK, Beyrau R, Braun S, et al. Multicenter analytical evaluation of a high-sensitivity troponin $\mathrm{T}$ assay. Clin Chim Acta 2011 Jan 8 .

14. Agewall S, Giannitsis E, Jernberg T, Katus H. Troponin elevation in coronary vs. non-coronary disease. Eur Heart J 2011 Feb; 32(4):404-11.

15. Januzzi JL, Jr., Bamberg F, Lee H, et al. High-sensitivity troponin $\mathrm{T}$ concentrations in acute chest pain patients evaluated with cardiac computed tomography. Circulation 2010 Mar 16;121 (10):1227-34

16. Javed U, Aftab W, Ambrose JA, et al. Frequency of elevated troponin I and diagnosis of acute myocardial infarction. Am J Cardiol 2009 Jul 1;104(1):9-13.

17. Agewall S, Eurenius L, Hofman-Bang C, et al. Myocardial infarction with angiographically normal coronary arteries. Atherosclerosis 2011 May 6. doi:10.1016/j.atherosclerosis. 2011.04 036 .

18. Giannitsis E, Kurz K, Hallermayer K, Jarausch J, Jaffe AS, Katus HA. Analytical validation of a high-sensitivity cardiac troponin T assay. Clin Chem 2010 Feb;56(2):254-61.
19. Jaffe AS, Apple FS, Morrow DA, Lindahl B, Katus HA. Being rational about (im)precision: a statement from the Biochemistry Subcommittee of the Joint European Society of Cardiology/American College of Cardiology Foundation/American Heart Association/World Heart Federation Task Force for the definition of myocardial infarction. Clin Chem 2010 Jun;56(6):941-3.

20. Aakre KM, Sandberg S. Can changes in troponin results be useful in diagnosing myocardial infarction? Clin Chem 2010 Jul;56 (7):1047-9.

21. Wu AH, Jaffe AS, Apple FS, et al. National Academy of Clinical Biochemistry laboratory medicine practice guidelines: use of cardiac troponin and B-type natriuretic peptide or $\mathrm{N}$-terminal proB-type natriuretic peptide for etiologies other than acute coronary syndromes and heart failure. Clin Chem 2007 Dec;53 (12):2086-96.

22. Eggers KM, Jaffe AS, Venge P, Lindahl B. Clinical implications of the change of cardiac troponin I levels in patients with acute chest pain - an evaluation with respect to the Universal Definition of Myocardial Infarction. Clin Chim Acta 2011 Jan 14;412 $(1-2): 91-7$.

23. Reichlin T, Irfan A, Twerenbold R, et al. Utility of absolute and relative changes in cardiac troponin concentrations in the early diagnosis of acute myocardial infarction. Circulation $2011 \mathrm{Jul}$ $12 ; 124(2): 136-45$

24. Thygesen K, Mair J, Katus H, et al. Recommendations for the use of cardiac troponin measurement in acute cardiac care. Eur Heart J 2010 Sep;31(18):2197-204.

25. Wu AH. Interpretation of high sensitivity cardiac troponin I results: reference to biological variability in patients who present to the emergency room with chest pain: case report series. Clin Chim Acta 2009 Mar;401(1-2):170-4.

26. Frankenstein L, Wu AH, Hallermayer K, Wians FH, Jr., Giannitsis E, Katus HA. Biological Variation and Reference Change Value of High-Sensitivity Troponin $\mathrm{T}$ in Healthy Individuals during Short and Intermediate Follow-up Periods. Clin Chem 2011 Epub Apr 25.

27. Kavsak PA, MacRae AR, Lustig V, et al. The impact of the ESC/ACC redefinition of myocardial infarction and new sensitive troponin assays on the frequency of acute myocardial infarction. Am Heart J 2006 Jul;152(1):118-25.

28. Apple FS, Pearce LA, Smith SW, Kaczmarek JM, Murakami MM. Role of monitoring changes in sensitive cardiac troponin I assay results for early diagnosis of myocardial infarction and prediction of risk of adverse events. Clin Chem 2009 May;55 (5):930-7.

\section{Correspondence:}

Trygve Sørdahl Hall

Department of Cardiology

Lovisenberg Diakonale Hospital

Lovisenberggata 17

0440 Oslo, Norway

Tel.: $\quad+4747500700$

Fax: +472322565

Email: tshall@online.no 